\title{
Measurement of the strange quark helicity distribution from semi-inclusive DIS on the deuteron
}

\author{
Markus Ehrenfried \\ for the HERMES Collaboration
}

JUSTUS-LIEBIG-

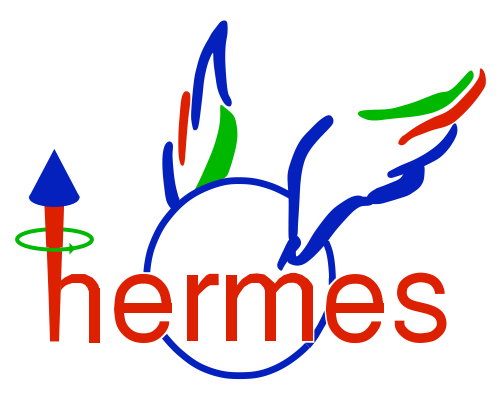




\section{Disentangling the contributions to the nucleon's spin}

$$
\left\langle s_{z}^{N}\right\rangle=\frac{1}{2}=\frac{1}{2} \Delta \Sigma+L_{z}^{q}+\Delta G+L_{z}^{G}
$$
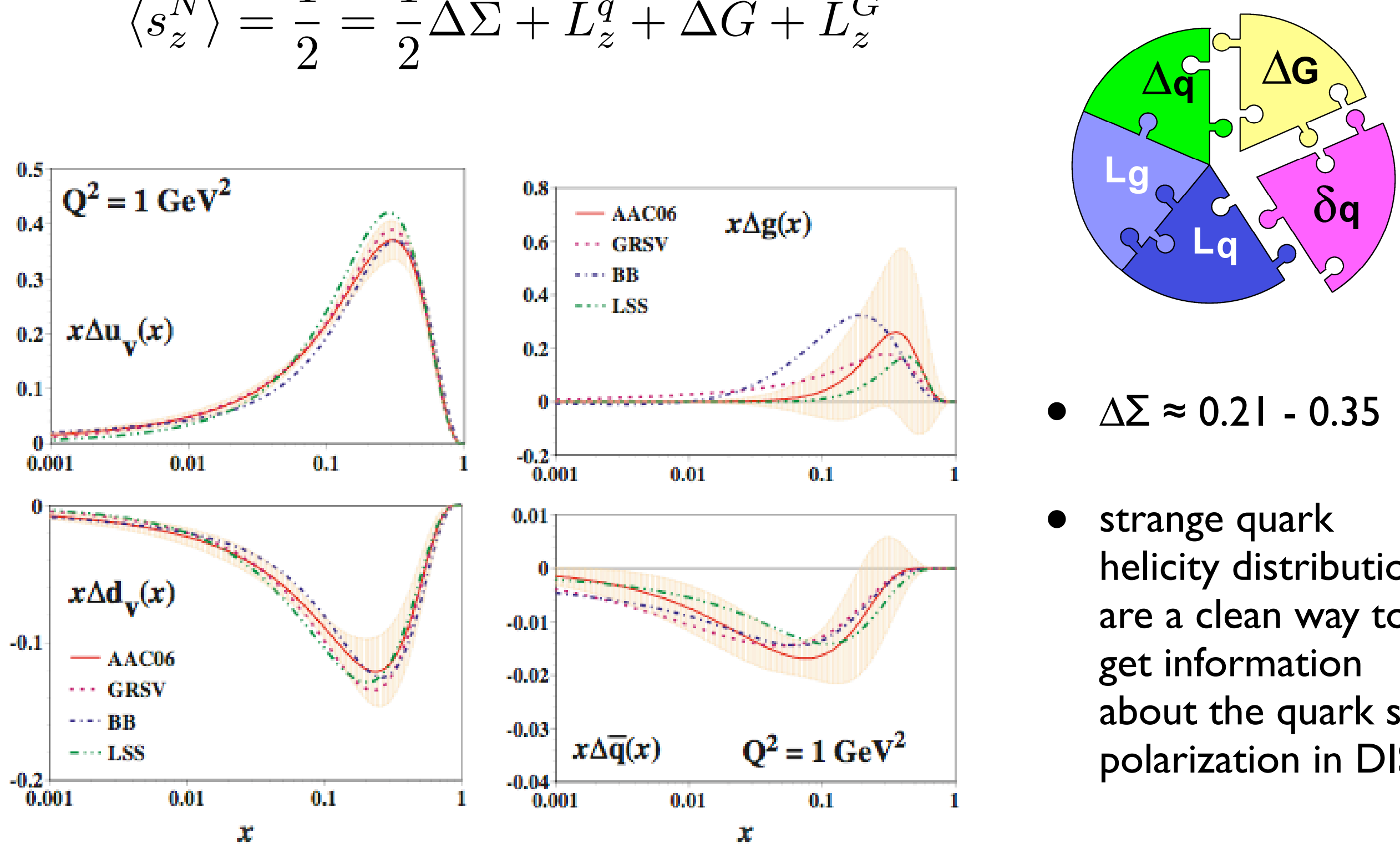

- $\Delta \Sigma \approx 0.21-0.35$

- strange quark helicity distributions are a clean way to get information about the quark sea polarization in DIS.

AAC: M. Hirai et al., hep-ph/06032I3 (2006) 
Disentangling the contributions to the nucleon's spin

$$
\left\langle s_{z}^{N}\right\rangle=\frac{1}{2}=\frac{1}{2} \Delta \Sigma+L_{z}^{q}+\Delta G+L_{z}^{G}
$$
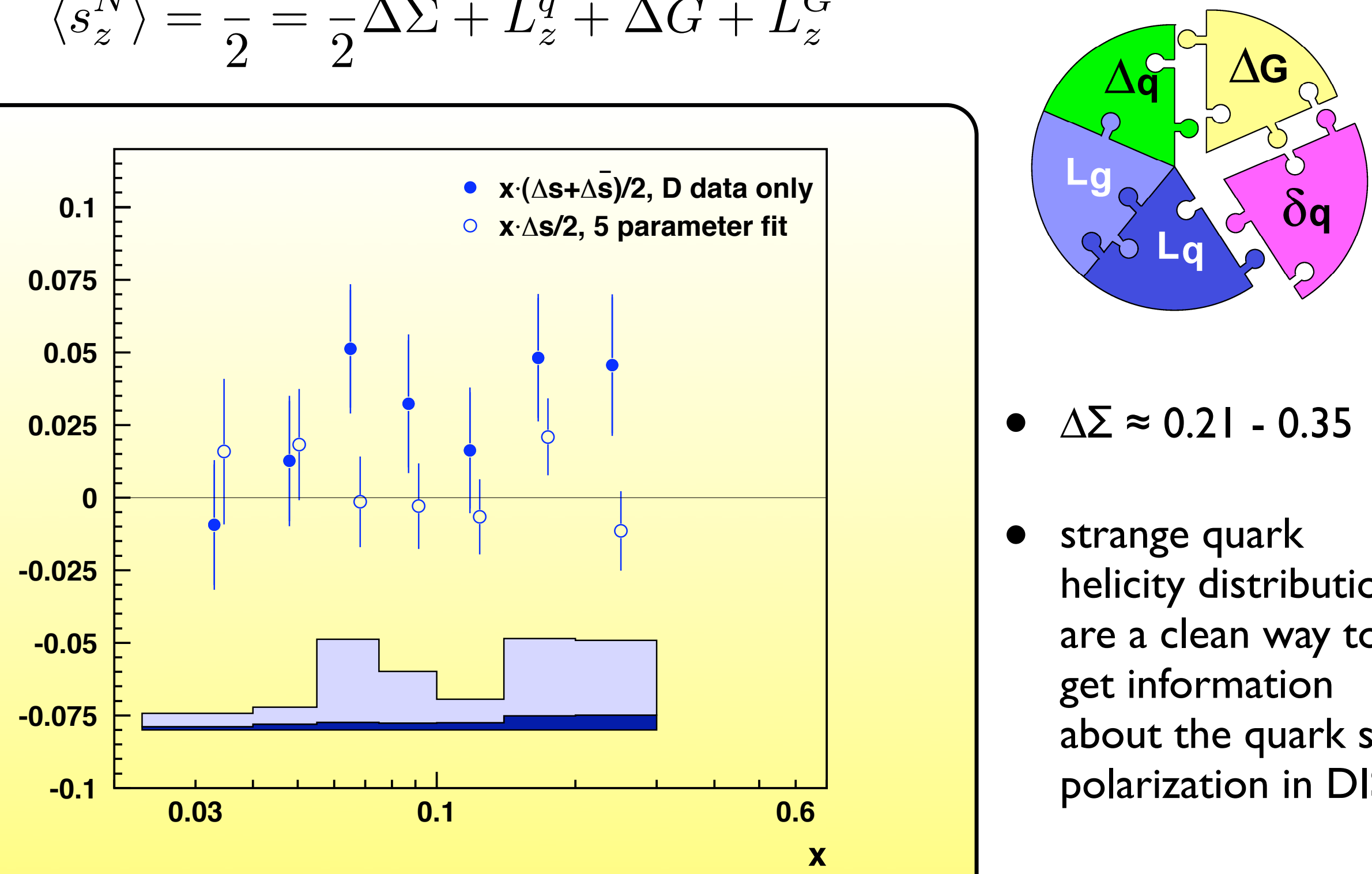

- $\Delta \Sigma \approx 0.21-0.35$

- strange quark helicity distributions are a clean way to get information about the quark sea polarization in DIS.

A. Airpetian et al. (HERMES), Phys. Rev. D 7I, 0 I 2003 (2005) 


\section{Semi-inclusive DIS}

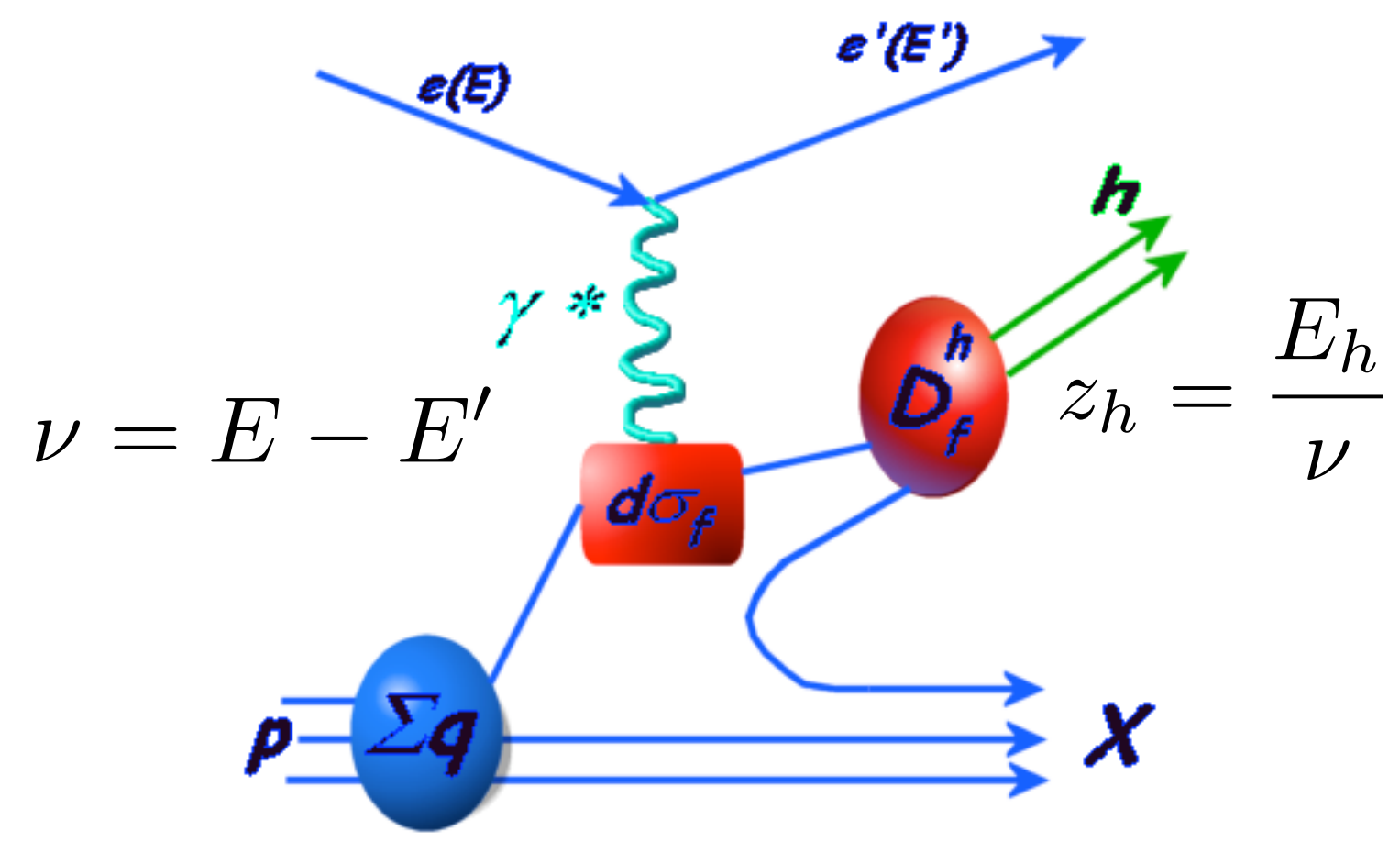

- observation of a coincindent hadron in the final state

- flavour structure of the hadron is correlated to the quark which was struck inside the nucleon

- this analysis:

$$
\begin{aligned}
x & =\frac{Q^{2}}{2 M \nu} \\
Q^{2} & =-q^{2} \stackrel{\text { lab. }}{\simeq} 4 E E^{\prime} \sin ^{2} \frac{\theta}{2}
\end{aligned}
$$

$K^{ \pm}$double spin asymmetry on an deuteron target are used to probe the strange quark sea. 


\section{"Isoscalar" measurement of $\Delta s+\Delta \bar{s}$}

- strange quarks carry no isospin

$I I m$ the strange seas in the proton and the neutron are identical

- the deuteron is an isoscalar target

$M$ fragmentation process in DIS can be described without assumptions regarding isospin dependent fragmentation

- "fragmentation functions" needed in this analysis are obtained from multiplicities directly at HERMES kinematics with the same data

- symmetry assumptions:

(i) isospin symmetry between proton and neutron

(ii) charge-conjugation invariance in fragmentation

- spin asymmetries used: $A_{1, d}^{K^{ \pm}}\left(x, Q^{2}, z\right)$ and $A_{1, d}\left(x, Q^{2}\right)$ 
Semi-inclusive virtual photo-absorption cross section for kaon production (in LO):

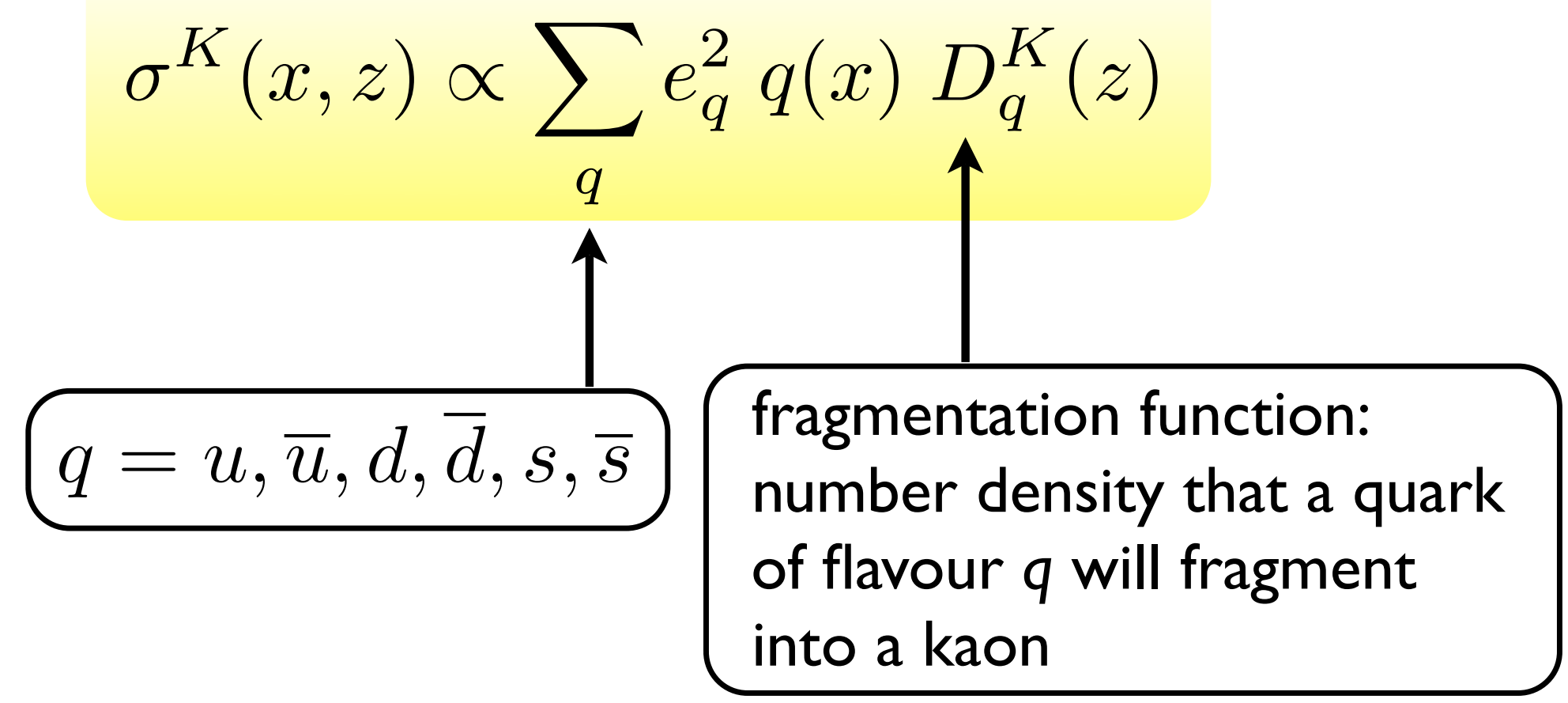

Double-spin asymmetry:

$$
A_{1}^{K}(x, z)=\frac{\sigma_{1 / 2}^{K}-\sigma_{3 / 2}^{K}}{\sigma_{1 / 2}^{K}+\sigma_{3 / 2}^{K}}=\frac{\sum_{q} e_{q}^{2} \Delta q(x) D_{q}^{K}(z)}{\sum_{q} e_{q}^{2} q(x) D_{q}^{K}(z)}
$$


Asymmetry expressed with purities: $\quad A_{1}^{K^{ \pm}}(x, z)=\sum_{q} \mathcal{P}_{q}^{K^{ \pm}}(x, z) \frac{\Delta q(x)}{q(x)}$ with $\mathcal{P}_{q}^{K^{ \pm}}(x, z)=\frac{e_{q}^{2} q(x) D_{q}^{K^{ \pm}}(z)}{\sum_{q^{\prime}} e_{q^{\prime}}^{2} q^{\prime}(x) D_{q^{\prime}}^{K^{ \pm}}(z)}$ "Probability that the virtual photon struck a quark of flavour $q$ in the nucleon when a $K^{ \pm}$is detected."

Simple linear relationship between the two measured asymmetries and the total non-strange quark distribution $Q(x) \equiv u(x)+\bar{u}(x)+d(x)+\bar{d}(x)$ and total strange quark distribution $S(x) \equiv s(x)+\bar{s}(x)$ :

$$
\left(\begin{array}{c}
A_{1, d}(x) \\
A_{1, d}^{K^{ \pm}}(x)
\end{array}\right) \propto\left(\begin{array}{cc}
\mathcal{P}_{Q}(x) & \mathcal{P}_{S}(x) \\
\mathcal{P}_{Q}^{K^{ \pm}}(x) & \mathcal{P}_{S}^{K^{ \pm}}(x)
\end{array}\right)\left(\begin{array}{c}
\Delta Q(x) / Q(x) \\
\Delta S(x) / S(x)
\end{array}\right)
$$




$$
\left(\begin{array}{c}
A_{1, d}(x) \\
A_{1, d}^{K^{ \pm}}(x)
\end{array}\right) \propto\left(\begin{array}{cc}
\mathcal{P}_{Q}(x) & \mathcal{P}_{S}(x) \\
\mathcal{P}_{Q}^{K^{ \pm}}(x) & \mathcal{P}_{S}^{K^{ \pm}}(x)
\end{array}\right)\left(\begin{array}{c}
\Delta Q(x) / Q(x) \\
\Delta S(x) / S(x)
\end{array}\right)
$$

Purities in terms of PDFs and fragmentation functions:

$$
\begin{aligned}
& \mathcal{P}_{Q}(x)=\frac{5 Q(x)}{5 Q(x)+2 S(x)} \quad \mathcal{P}_{S}(x)=\frac{2 S(x)}{5 Q(x)+2 S(x)} \\
& \mathcal{P}_{Q}^{K^{ \pm}}(x)=\frac{Q(x) \int \mathcal{D}_{\text {non-strange }}^{K^{ \pm}}(z) \mathrm{d} z}{Q(x) \int \mathcal{D}_{\text {non-strange }}^{K^{ \pm}}(z) \mathrm{d} z+S(x) \int \mathcal{D}_{\text {strange }}^{K^{ \pm}}(z) \mathrm{d} z} \\
& \mathcal{P}_{S}^{K^{ \pm}}(x)=\frac{S(x) \int \mathcal{D}_{\text {strange }}^{K^{ \pm}}(z) \mathrm{d} z}{Q(x) \int \mathcal{D}_{\text {non-strange }}^{K^{ \pm}}(z) \mathrm{d} z+S(x) \int \mathcal{D}_{\text {strange }}^{K^{ \pm}}(z) \mathrm{d} z}
\end{aligned}
$$

with

$$
\begin{gathered}
\int \mathcal{D}_{\text {non-strange }}^{K^{ \pm}}(z) \mathrm{d} z=4 \int D_{\mathrm{u}}^{K^{ \pm}}(z) \mathrm{d} z+\int D_{\mathrm{d}}^{K^{ \pm}}(z) \mathrm{d} z \\
\int \mathcal{D}_{\text {strange }}^{K^{ \pm}}(z) \mathrm{d} z=2 \int D_{\mathrm{s}}^{K^{ \pm}}(z) \mathrm{d} z
\end{gathered}
$$


These fragmentation functions

$$
\begin{gathered}
\int \mathcal{D}_{\text {non-strange }}^{K^{ \pm}}(z) \mathrm{d} z=4 \int D_{\mathrm{u}}^{K^{ \pm}}(z) \mathrm{d} z+\int D_{\mathrm{d}}^{K^{ \pm}}(z) \mathrm{d} z \\
\int \mathcal{D}_{\text {strange }}^{K^{ \pm}}(z) \mathrm{d} z=2 \int D_{\mathrm{s}}^{K^{ \pm}}(z) \mathrm{d} z
\end{gathered}
$$

can be obtained from unpolarized SIDIS data taken at HERMES kinematics, assuming only charge-conjugation invariance in the fragmentation process:

$$
D_{u}^{K^{ \pm}}=D_{\bar{u}}^{K^{\mp}}, \quad D_{d}^{K^{ \pm}}=D_{\frac{K^{\mp}}{}}^{\mp} \quad D_{s}^{K^{ \pm}}=D_{\bar{s}}^{K^{\mp}}
$$

The strange and non-strange fragmentation functions are then extracted by fitting the $x$ dependence of this ratio:

$$
\frac{\mathrm{d} N^{K^{ \pm}}(x) / \mathrm{d} x}{\mathrm{~d} N^{\mathrm{DIS}}(x) / \mathrm{d} x}=\frac{Q(x) \int \mathcal{D}_{\text {non-strange }}^{K^{ \pm}}(z) \mathrm{d} z+S(x) \int \mathcal{D}_{\text {strange }}^{K^{ \pm}}(z) \mathrm{d} z}{5 Q(x)+2 S(x)}
$$




$$
\frac{\mathrm{d} N^{K^{ \pm}}(x) / \mathrm{d} x}{\mathrm{~d} N^{\operatorname{DIS}}(x) / \mathrm{d} x}=\frac{Q(x) \int \mathcal{D}_{\text {non-strange }}^{K^{ \pm}}(z) \mathrm{d} z+S(x) \int \mathcal{D}_{\text {strange }}^{K^{ \pm}}(z) \mathrm{d} z}{5 Q(x)+2 S(x)}
$$

\begin{tabular}{|lllll|}
\hline & This Work & Kretzer KKP \\
$\int D_{n s t r g}^{K}(z) d z$ & $0.379 \pm 0.002 \pm 0.009$ & 1.103 & 1.111 \\
$\int D_{s t r g}^{K}(z) d z$ & $1.722 \pm 0.024 \pm 0.108$ & 0.783 & 0.296 \\
\hline
\end{tabular}

Strangeness suppression factor for $s \bar{s}$ production is important for $\mathcal{D}_{\text {non-strange }}^{K^{ \pm}}(z)$

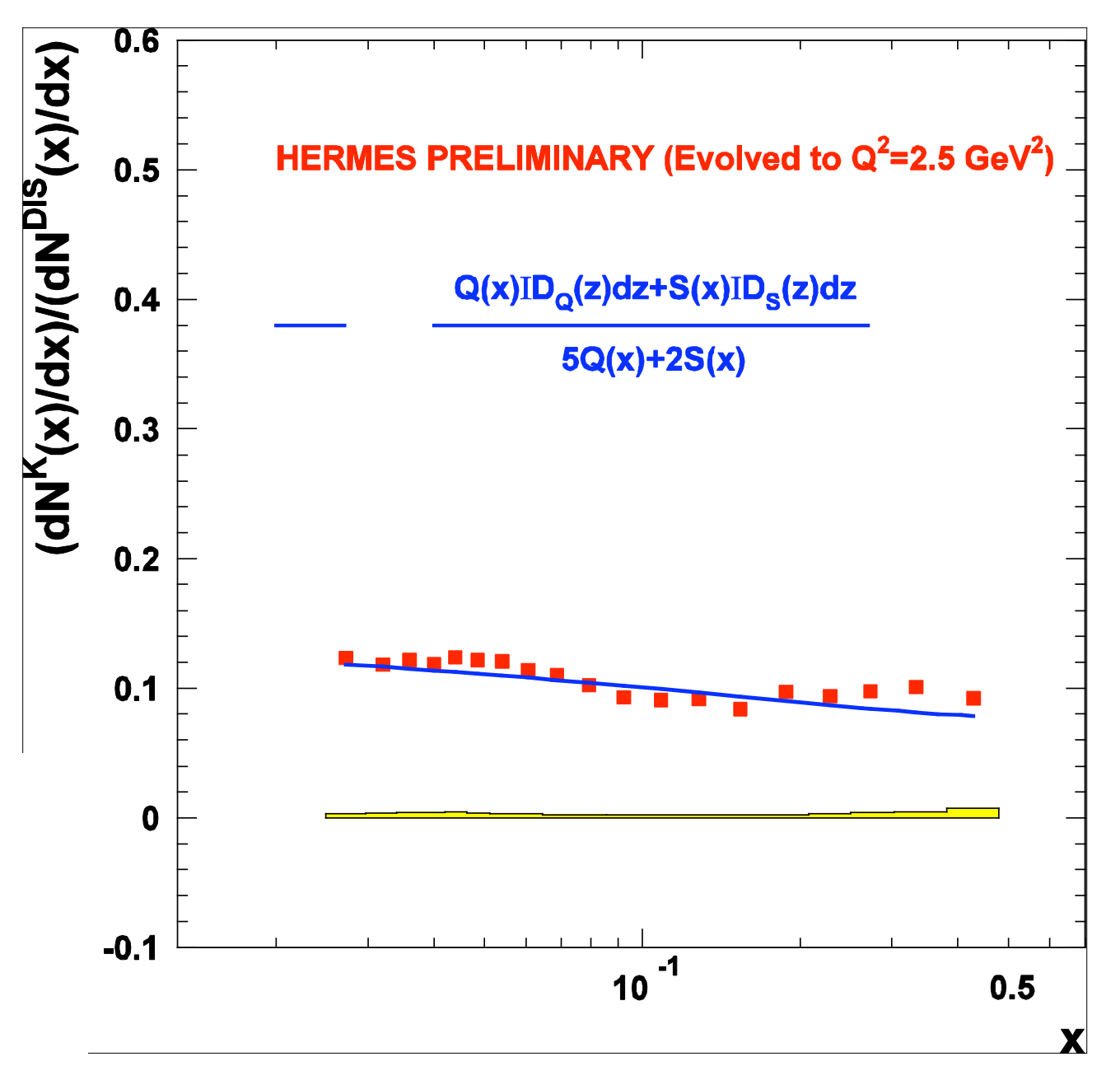




\section{Event selection}
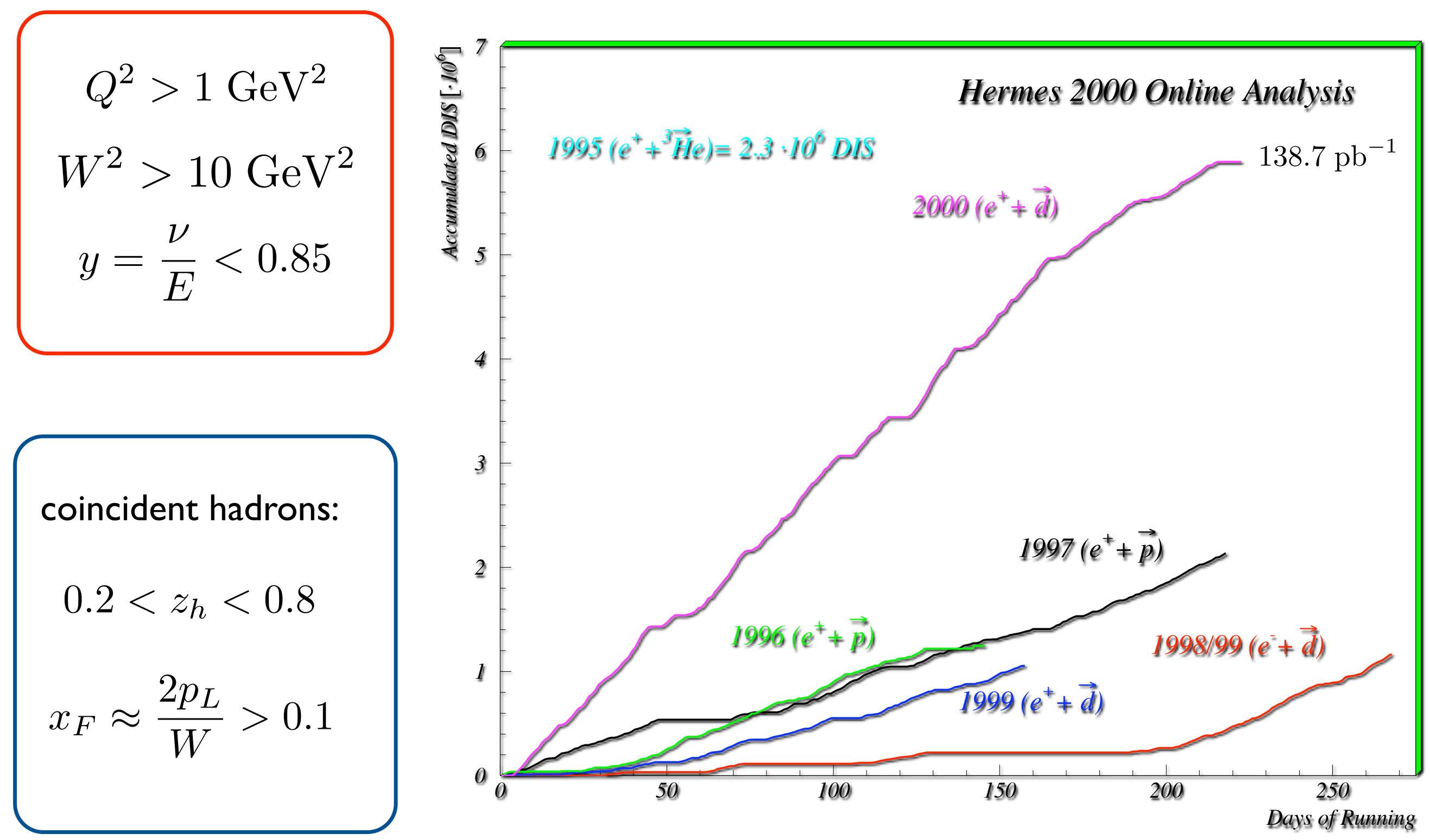

$p_{L}=$ longitudinal momentum of hadron w.r.t. the $\gamma^{\star}$ 
$\left(\begin{array}{c}A_{1, d}(x) \\ A_{1, d}^{K_{1}}(x)\end{array}\right) \propto\left(\begin{array}{cc}\mathcal{P}_{Q}(x) & \mathcal{P}_{S}(x) \\ \mathcal{P}_{Q}^{K^{ \pm}}(x) & \mathcal{P}_{S}^{K^{ \pm}}(x)\end{array}\right)\left(\begin{array}{c}\Delta Q(x) / Q(x) \\ \Delta S(x) / S(x)\end{array}\right)$

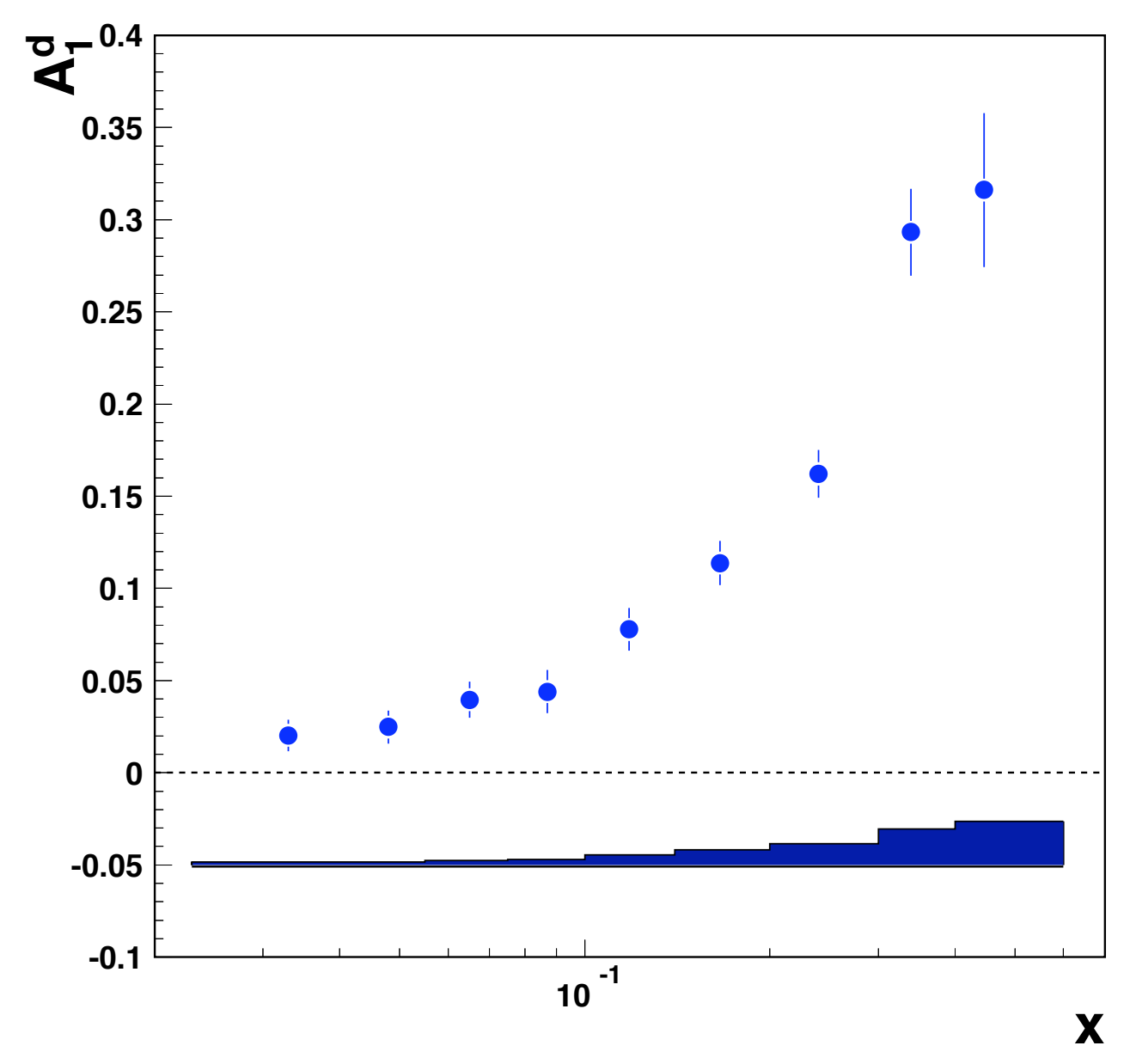

inclusive asymmetry

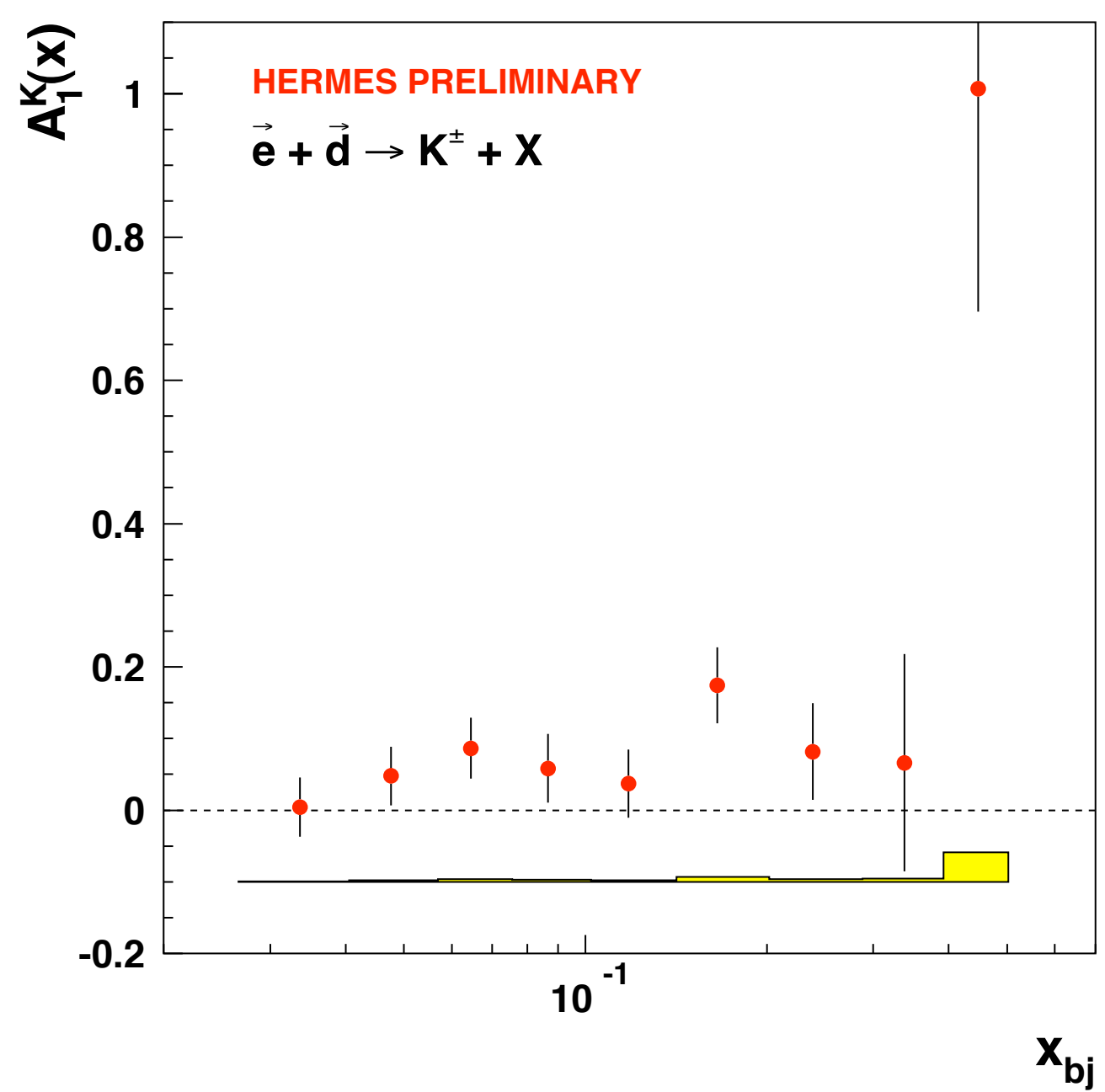

Kaon asymmetry 


$$
\left(\begin{array}{c}
A_{1, d}(x) \\
A_{1, d}^{K^{ \pm}}(x)
\end{array}\right) \propto\left(\begin{array}{cc}
\mathcal{P}_{Q}(x) & \mathcal{P}_{S}(x) \\
\mathcal{P}_{Q}^{K^{ \pm}}(x) & \mathcal{P}_{S}^{K^{ \pm}}(x)
\end{array}\right)\left(\begin{array}{c}
\Delta Q(x) / Q(x) \\
\Delta S(x) / S(x)
\end{array}\right)
$$

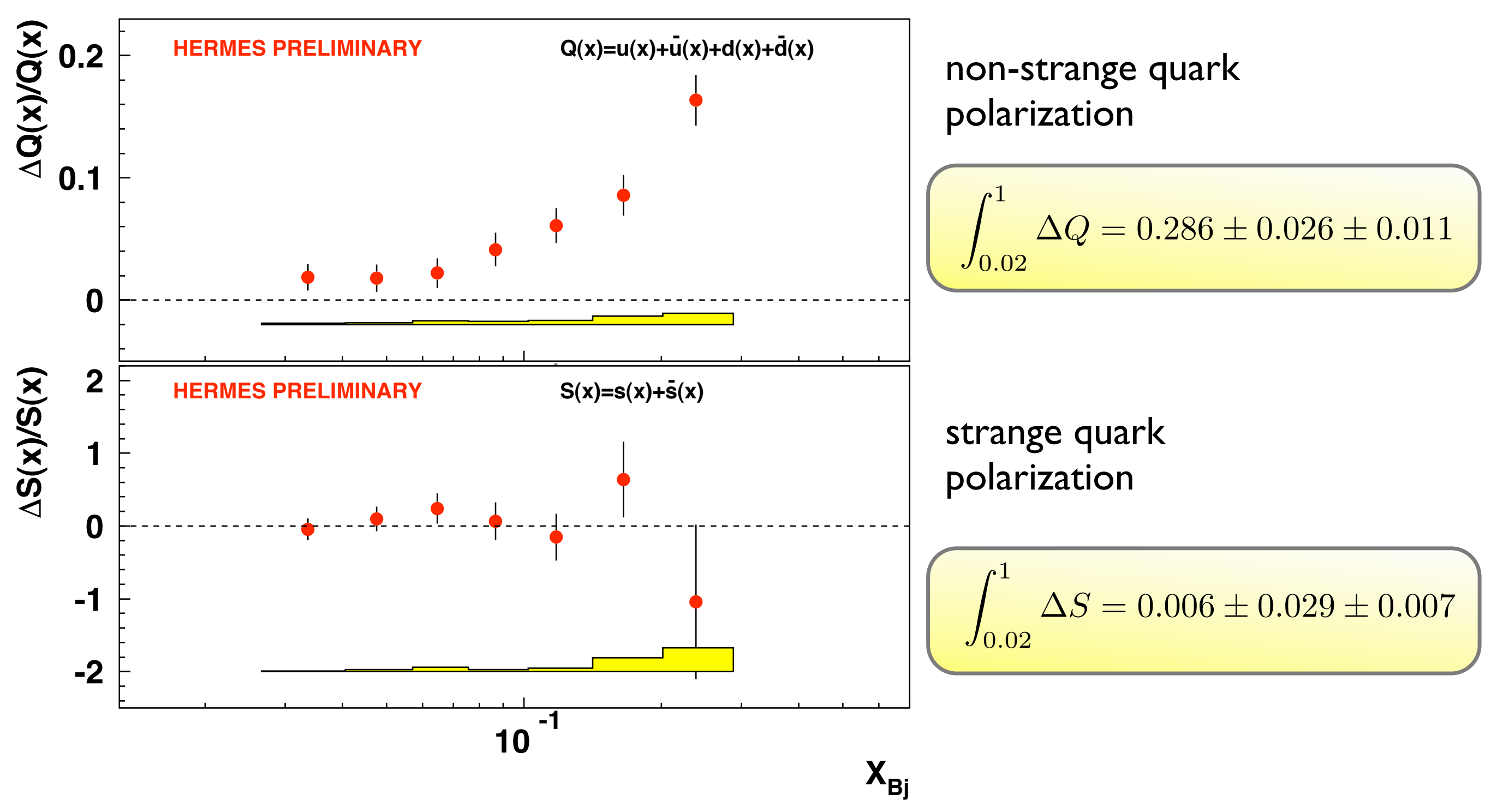




\section{Summary}

$$
\int_{0.02}^{1} \Delta S=0.006 \pm 0.029 \pm 0.007
$$

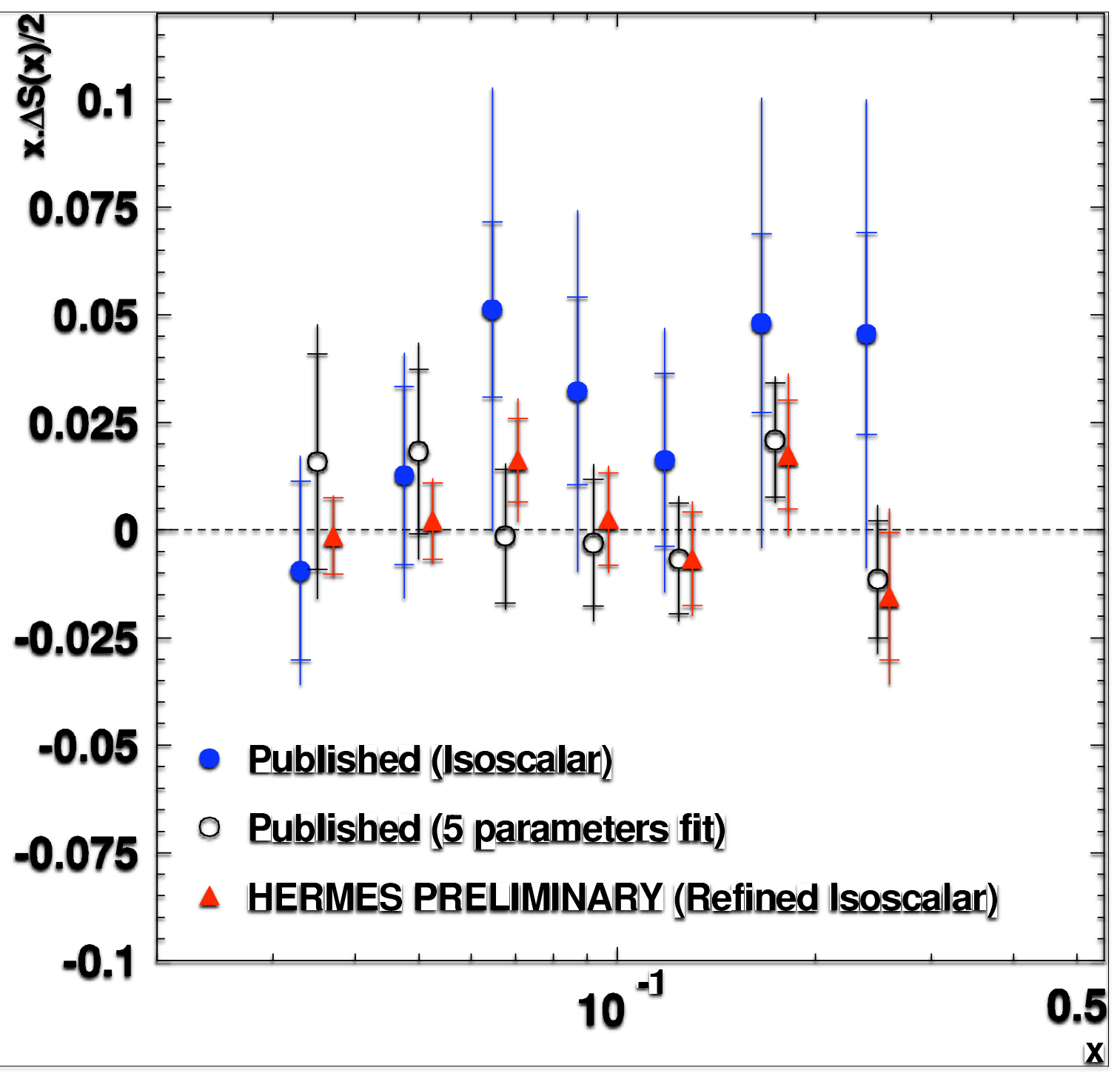

- $\Delta \mathrm{S}$ is consistent with zero within the measured range

$0.02<x<0.60$

$0.2<z<0.8$

- First moment of $\Delta S$ is zero within uncertainties. 